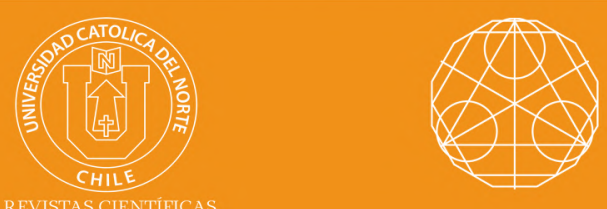

\title{
Note on extended hypergeometric function
}

\author{
R. Jana* iD orcid.org/0000-0001-5017-4189 \\ B. Maheshwari** \\ A. Shukla** iD orcid.org/0000-0002-2713-2017
}

*Sardar Vallabhbhai National Institute of Technology, Dept. of Applied Mathematics \& Humanities, Surat, GJ, India. $\nabla$ rkjana2003@yahoo.com

${ }^{* *}$ Sardar Vallabhbhai National Institute of Technology , Dept. of Applied Mathematics \& Humanities, Surat, GJ, India. $\nabla$ bhumi0512@gmail.com

${ }^{* * *}$ Sardar Vallabhbhai National Institute of Technology, Dept. of Applied Mathematics \& Humanities, Surat, GJ, India. $\square$ ajayshukla2@rediffmail.com

Received: May 2018 | Accepted: July 2018

\section{Abstract:}

In this paper, we present an extension of the classical hypergeometric functions using extended gamma function due to Jumarie and obtained some properties.

Keywords: Gamma function; Pochhammer symbols; Hypergeometric functions; Integral transforms; Fractional calculus.

MSC (2010): 33B15, 33C05, 33C20, 44A05, 26 A33.

\section{Cite this article as (IEEE citation style):}

R. Jana, B. Maheshwariand and A. Shukla, "Note on extended hypergeometric function", Proyecciones (Antofagasta, On line), vol. 38, no. 3, pp. 585-595, Aug. 2019, doi: 10.22199/issn.0717-62792019-03-0037 [Accessed dd-mm-yyyy].

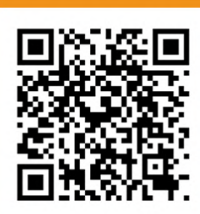

Article copyright: (c) 2019 Ranjan Kumar Jana, Bhumika Maheshwari and Ajay Kumar Shukla. This is an open access article distributed under the terms of the Creative Commons Licence, which permits unrestricted use and distribution provided the original author and source are credited. 


\section{Introduction}

In 2006, Jumarie [4] gave an interesting extension of the gamma function as

$$
\Gamma_{\alpha}(x)=(\alpha !)^{-1} \int_{0}^{\infty} E_{\alpha}\left(-t^{\alpha}\right) t^{(x-1) \alpha}(d t)^{\alpha}, \quad 0<\alpha<1,
$$

where $E_{\alpha}(u)$ is the Mittag-Leffler function (1.2)

$$
E_{\alpha}(u)=\sum_{k=0}^{\infty} \frac{u^{k}}{(\alpha k) !}, \quad \alpha \in \mathbf{C} .
$$

Here $(\alpha !)$ denotes $\Gamma(\alpha+1)$. We use this notation throughout the paper.

The integral involved in (1.1) is the fractional integral with respect to $(d t)^{\alpha}$ given by Jumarie (see, for details [3], [4], [5], [7], [8], [9], [10], [11]). This modified integral has the following connection via fractional calculus, with the usual integral [5]

$$
\int_{0}^{x} f(\xi)(d \xi)^{\alpha}=\alpha \int_{0}^{x}(x-\xi)^{\alpha-1} f(\xi) d \xi .
$$

We define the extension of Gauss hypergeometric function as follows

$$
{ }_{2}^{\alpha} F_{1}(a, b ; c ; z)=\sum_{n=0}^{\infty} \frac{(a)_{n, \alpha}(b)_{n}}{(c)_{n}} \frac{z^{n}}{n !},
$$

where $0<\alpha \leq 1, a, b, c \in \mathbf{C}$ with $c$ is never zero or negative integer.

Here $(\lambda)_{n, \alpha}$ is the extended Pochhammer symbol defined by

$$
(\lambda)_{n, \alpha}=\frac{\Gamma_{\alpha}(\lambda+v)}{\Gamma_{\alpha}(\lambda)}
$$

where $\lambda \in \mathbf{C}, n \in \mathbf{N} \cup\{0\}, 0<\alpha \leq 1$.

In the definition (1.5), $\Gamma_{\alpha}(\lambda)$ is the extended gamma function given by (1.1). 


\section{Generalized integral transforms}

In 2012, Virchenko [16] introduced the following generalizations of classical integral transforms.

Definition: The generalized Laplace integral transform

$$
L_{\gamma_{1}, \gamma_{2}}\{f(x) ; y\}=\int_{0}^{\infty} x^{\gamma_{2}} e^{-(x y)^{\gamma_{1}}} f(x) d x
$$

and

$\tilde{L}_{\gamma_{1}, \gamma_{2}, \gamma}\{f(x) ; y\}=\int_{0}^{\infty} x^{\gamma_{2}} e^{-(x y)^{\gamma_{1}}}{ }_{1} \Phi_{1}^{\tau, \beta}\left(\alpha ; c ;-b(x y)^{\gamma \gamma_{1}}\right) f(x) d x$,

where $x>0, \gamma \in C, \gamma_{1}>0, \gamma_{2}>0, b \geq 0,{ }_{1} \Phi_{1}^{\tau, \beta}$ is the $(\tau, \beta)$-generalized hypergeometric function $[15]$

$$
{ }_{1} \Phi_{1}^{\tau, \beta}\left(\alpha ; c ;-b(x y)^{\gamma \gamma_{1}}\right)=\frac{1}{B(a, c-a)} \int_{0}^{1} t^{a-1}(1-t)^{c-a-1}{ }_{1} \Psi_{1}\left[\begin{array}{c}
(c ; \tau) \\
(c ; \beta)
\end{array} \mid z t^{\tau}\right] d t
$$

$(\operatorname{Re}(\mathrm{c})>\operatorname{Re}(\mathrm{a})>0 ;\{\tau, \beta\} \subset R ; \tau>0 ; \tau-\beta<1)$

and ${ }_{p} \Psi_{q}$ is the generalized hypergeometric Wright function

$$
\begin{gathered}
{ }_{p} \Psi_{q}\left[\begin{array}{c}
\left(a_{1} ; \alpha_{1}\right) \ldots\left(a_{p} ; \alpha_{p}\right) \\
\left(b_{1} ; \beta_{1}\right) \ldots\left(b_{q} ; \beta_{q}\right)
\end{array} \mid z\right]=\sum_{n=0}^{\infty} \frac{\prod_{i=0}^{p} \Gamma\left(a_{i}+n \alpha_{i}\right)}{\prod_{j=0}^{q} \Gamma\left(b_{j}+n \beta_{j}\right)} \frac{z^{n}}{n !}, \\
\left(z \in C ; a_{i}, b_{j} \in C ;\left\{\alpha_{i}, \beta_{j}\right\} \subset R ; a_{i}, b_{j} \neq 0 ; 1+\sum_{j=1}^{q} \beta_{j}-\sum_{i=1}^{p} \alpha_{i} \geq 0 .\right.
\end{gathered}
$$

Definition: The generalized Stieltjes integral transform

$$
\begin{aligned}
& P_{1}^{\gamma_{1}, \gamma_{2}, \gamma_{3}, \gamma_{4}}\{f(u) ; x\}=\tilde{P}_{1}\{f(u) ; x\} \\
& =\frac{\Gamma(c)}{\Gamma\left(a_{1}\right) \Gamma\left(a_{2}\right)} \int_{0}^{\infty} \frac{u^{\gamma_{2}} f(u)}{\left(x^{\gamma_{1}}+u^{\gamma_{1}}\right)^{\gamma_{3}}} 2 \Psi_{1}\left[\begin{array}{c|c}
\left(a_{1} ; \tau\right) ;\left(a_{2} ; \gamma\right) & \left.-b\left(\frac{u^{\gamma_{1}}}{x^{\gamma_{1}}+u^{\gamma_{1}}}\right)^{\gamma_{4}}\right] d u
\end{array}\right]
\end{aligned}
$$


and

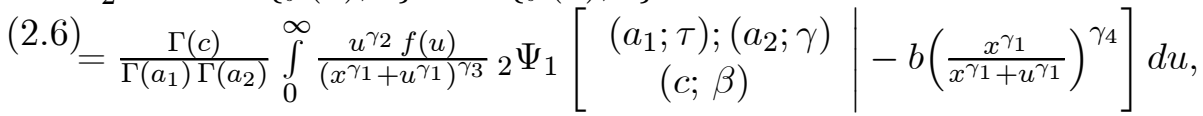

where $\operatorname{Re}\left(a_{1}\right)>0, \operatorname{Re}\left(a_{2}\right)>0, \operatorname{Re}(c)>0, \gamma_{i}>0 ;\{\tau, \beta\} \subset R ; \tau>$ $0 ; \tau-\beta<1 ; b \geq 0$ and ${ }_{2} \Psi_{1}$ is defined by $(2.3)$.

Theorem 1. If $0<x<1, \gamma \in C, \gamma_{1}>0, \gamma_{2}>0, b \geq 0 ; \alpha_{1}, \alpha_{2}, \rho \in$ $C ; \rho \notin\{0,-1,-2, \ldots\} ; 0<\alpha \leq 1$, then

$$
L_{\gamma_{1}, \gamma_{2}}\left\{{ }_{2}^{\alpha} F_{1}\left(\alpha_{1}, \alpha_{2} ; \rho ; x\right) ; y\right\}=\frac{1}{\gamma_{1}} y^{-\gamma_{2}-1} \sigma_{1}(y),
$$

where

$$
\sigma_{1}(y)=\sum_{n=0}^{\infty} \frac{\left(\alpha_{1}\right)_{n, \alpha}\left(\alpha_{2}\right)_{n}}{(\rho)_{n}} \Gamma\left(\frac{\gamma_{2}+n+1}{\gamma_{1}}\right) \frac{y^{-n}}{n !} .
$$

In particular when $\gamma_{1}=1$, then

$L_{1, \gamma_{2}}\left\{{ }_{2}^{\alpha} F_{1}\left(\alpha_{1}, \alpha_{2} ; \rho ; x\right) ; y\right\}=y^{-\gamma_{2}-1} \Gamma\left(\gamma_{2}+1\right){ }_{2}^{\alpha} F_{1}\left(\alpha_{1}, \alpha_{2}, \gamma_{2}+1 ; \rho ; \frac{1}{y}\right)$.

and

$$
\tilde{L}_{\gamma_{1}, \gamma_{2}, \gamma}\left\{{ }_{2}^{\alpha} F_{1}\left(\alpha_{1}, \alpha_{2} ; \rho ; x\right) ; y\right\}=\frac{1}{\gamma_{1}} \frac{\Gamma(c)}{\Gamma(a)} y^{-\gamma_{2}-1} \sigma_{2}(b, x),
$$

where

$$
\sigma_{2}(b, x)=\sum_{n=0}^{\infty} \frac{\left(\alpha_{1}\right)_{n}\left(\alpha_{2}\right)_{n}}{(\rho)_{n}}{ }_{2} \Psi_{1}\left[\begin{array}{c}
(a ; \tau) ;\left(\frac{\gamma_{2}+n+1}{\gamma_{1}} ; \gamma\right) \\
(c ; \beta)
\end{array} \mid-b\right] \frac{y^{-n}}{n !}
$$

Proof. From the linearity property of generalized Laplace transform (2.1) (see, [16]), one can write the following

$$
L_{\gamma_{1}, \gamma_{2}}\left\{{ }_{2}^{\alpha} F_{1}\left(\alpha_{1}, \alpha_{2} ; \rho ; x\right) ; y\right\}=\sum_{n=0}^{\infty} \frac{\left(\alpha_{1}\right)_{n, \alpha}\left(\alpha_{2}\right)_{n}}{(\rho)_{n}}\left[\int_{0}^{\infty} e^{-(x y)^{\gamma_{1}}} x^{\gamma_{2}+n} d x\right] \frac{1}{n !} .
$$


Let us evaluate the integral involved in (2.10). By taking the substitutions $x y=t$ and $t^{\gamma_{1}}=u$, we can get

$$
\begin{aligned}
& \int_{0}^{\infty} e^{-(x y)^{\gamma_{1}}} x^{\gamma_{2}+n} d x \\
& =y^{-\gamma_{2}-k-1} \int_{0}^{\infty} e^{-t^{\gamma_{1}}} t^{\gamma_{2}+k} d t \\
& =\frac{1}{\gamma_{1}} y^{-\gamma_{2}-n-1} \int_{0}^{\infty} e^{-u} u^{\left(\frac{\gamma_{2}+n+1}{\gamma_{1}}-1\right)} d u=\frac{1}{\gamma_{1}} y^{-\gamma_{2}-n-1} \Gamma\left(\frac{\gamma_{2}+n+1}{\gamma_{1}}\right),
\end{aligned}
$$

using this in (2.10), one can get the formula (2.7).

Assertion (2.9) can be derived by using the formula [16]

$$
\tilde{L}_{\gamma_{1}, \gamma_{2}, \gamma}\left\{x^{k} ; y\right\}=\frac{1}{\gamma_{1}} \frac{\Gamma(c)}{\Gamma(a)} y^{-\gamma_{2}-k-1}{ }_{2} \Psi_{1}\left[\begin{array}{c}
(a ; \tau) ;\left(\frac{\gamma_{2}+n+1}{\gamma_{1}} ; \gamma\right) \\
(c ; \beta)
\end{array} \mid-b\right],
$$

and applying similar method leads to the proof of (2.7).

Theorem 2. If $\operatorname{Re}\left(a_{1}\right)>0, \operatorname{Re}\left(a_{2}\right)>0, \operatorname{Re}(c)>0, \gamma_{i}>0 ;\{\tau, \beta\} \subset$ $R ; \tau>0 ; \tau-\beta<1 ; b \geq 0 ; \alpha_{1}, \alpha_{2}, \rho \in C ; \rho \notin\{0,-1,-2, \ldots\} ; 0<\alpha \leq 1$, then

$P_{1}^{\gamma_{1}, \gamma_{2}, \gamma_{3}, \gamma_{4}}\left\{{ }_{2}^{\alpha} F_{1}\left(\alpha_{1}, \alpha_{2} ; \rho ; x\right) ; y\right\}=\frac{1}{\gamma_{1}} \frac{\Gamma(c)}{\Gamma\left(a_{1}\right) \Gamma\left(a_{2}\right)} y^{-\gamma_{1} \gamma_{3}+\gamma_{2}+1} \sigma_{3}(b, x)$,

where

$$
\begin{aligned}
& \sigma_{3}(b, x) \\
& =\sum_{n=0}^{\infty} \frac{\left(\alpha_{1}\right)_{n}\left(\alpha_{2}\right)_{n} \Gamma\left(\gamma_{3}-\frac{\gamma_{2}+n+1}{\gamma_{1}}\right)}{(\rho)_{n}} \Psi_{2} \Psi_{2}\left[\begin{array}{l}
\left.\left(a_{1} ; \tau\right) ;\left(a_{2} ; \gamma\right)\left(\frac{\gamma_{2}+n+1}{\gamma_{1}} ; \gamma_{4}\right) \mid-b\right] \frac{y^{n}}{n !} \\
(c ; \beta) ;\left(\gamma_{3} ; \gamma_{4}\right)
\end{array}\right.
\end{aligned}
$$

and

$P_{1}^{\gamma_{1}, \gamma_{2}, \gamma_{3}, \gamma_{4}}\left\{{ }_{2}^{\alpha} F_{1}\left(\alpha_{1}, \alpha_{2} ; \rho ; x\right) ; y\right\}=\frac{1}{\gamma_{1}} \frac{\Gamma(c)}{\Gamma\left(a_{1}\right) \Gamma\left(a_{2}\right)} y^{-\gamma_{1} \gamma_{3}+\gamma_{2}+1} \sigma_{4}(b, x)$,

where

$$
\begin{aligned}
& \sigma_{4}(b, x) \\
& =\sum_{n=0}^{\infty} \frac{\left(\alpha_{1}\right)_{n}\left(\alpha_{2}\right)_{n} \Gamma\left(\frac{\gamma_{2}+n+1}{\gamma_{1}}\right)}{(\rho)_{n}}{ }_{3} \Psi_{2}\left[\begin{array}{l}
\left.\left(a_{1} ; \tau\right) ;\left(a_{2} ; \gamma\right)\left(\gamma_{3}-\frac{\gamma_{2}+n+1}{\gamma_{1}} ; \gamma_{4}\right) \mid-b\right] \frac{y^{n}}{n !} \\
(c ; \beta) ;\left(\gamma_{3} ; \gamma_{4}\right)
\end{array}\right.
\end{aligned}
$$


Proof. From the definition (2.5), we have

$$
\begin{aligned}
& P_{1}^{\gamma_{1}, \gamma_{2}, \gamma_{3}, \gamma_{4}} \quad\left\{{ }_{2}^{\alpha} F_{1}\left(\alpha_{1}, \alpha_{2} ; \rho ; x\right) ; y\right\}=\frac{\Gamma(c)}{\Gamma\left(a_{1}\right) \Gamma\left(a_{2}\right)} \sum_{n=0}^{\infty} \frac{\left(\alpha_{1}\right)_{n}\left(\alpha_{2}\right)_{n}}{(\rho)_{n}}
\end{aligned}
$$

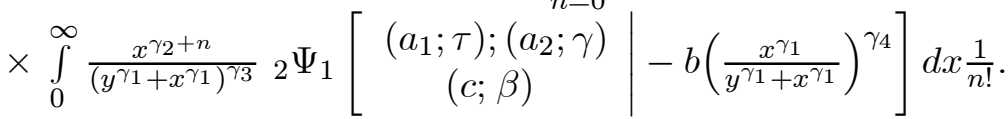

From (2.3) and changing the variable from $\frac{x}{y}=t$ and then $t^{\gamma_{1}}=u$, the integral appeared in (2.14) becomes

$$
\begin{aligned}
& \int_{0}^{\infty} \frac{x^{\gamma_{2}+n}}{\left(y^{\left.\gamma_{1}+x^{\gamma_{1}}\right)^{\gamma_{3}}}{ }_{2}\right.} \Psi_{1}\left[\begin{array}{c}
\left(a_{1} ; \tau\right) ;\left(a_{2} ; \gamma\right) \\
(c ; \beta)
\end{array} \mid-b\left(\frac{x^{\gamma_{1}}}{\left.\left.y^{\gamma_{1}+x^{\gamma_{1}}}\right)^{\gamma_{4}}\right] d x}\right.\right. \\
& =\frac{y^{-\gamma_{1} \gamma_{3}+\gamma_{2}+n+1}}{\gamma_{1}} \sum_{k=0}^{\infty} \frac{\Gamma\left(a_{1}+\tau k\right) \Gamma\left(a_{2}+\gamma k\right)}{\Gamma(c+\beta k)} \int_{0}^{\infty} u\left(\frac{\gamma_{2}+n+1}{\gamma_{1}}+\gamma_{4} k-1\right)(1+u)^{-\gamma_{3}-k \gamma_{4}} d u \frac{(-b)^{k}}{k !} \\
& =\frac{y^{-\gamma_{1} \gamma_{3}+\gamma_{2}+n+1}}{\gamma_{1}} \sum_{k=0}^{\infty} \frac{\Gamma\left(a_{1}+\tau k\right) \Gamma\left(a_{2}+\gamma k\right) \Gamma\left(\frac{\gamma_{2}+n+1}{\gamma_{1}}+\gamma_{4} k-1\right) \Gamma\left(\gamma_{3}-\frac{\gamma_{2}+n+1}{\gamma_{1}}\right)}{\Gamma(c+\beta k) \Gamma\left(\gamma_{3}+\gamma_{4} k\right)} \frac{(-b)^{k}}{k !} .
\end{aligned}
$$

Putting this in (2.14), we can reach the assertion (2.12).

Using the similar method, one can derive formula (2.13).

\section{Generalized fractional operators and ${ }_{p}^{\alpha} F_{q}(a, b ; c ; z)$}

The generalized fractional integral operators involving the Appell's function due to Saigo and Maeda [13] are defined by

$$
\begin{aligned}
& \left(I_{0+}^{\delta, \delta^{\prime}, \beta, \beta^{\prime}, \gamma} f\right)(x) \\
& =\frac{x^{-\delta}}{\Gamma(\gamma)} \int_{0}^{x}(x-t)^{\gamma-1} t^{-\delta^{\prime}} F_{3}\left(\delta, \delta^{\prime}, \beta, \beta^{\prime} ; \gamma ; 1-\frac{t}{x}, 1-\frac{x}{t}\right) f(t) d t \\
& \quad\left(\delta, \delta^{\prime}, \beta, \beta^{\prime}, \gamma \in \mathbf{C} \text { and } x>0 ; \operatorname{Re}(\gamma)>0\right)
\end{aligned}
$$

and

$$
\begin{gathered}
\left(I_{0-}^{\delta, \delta^{\prime}, \beta, \beta^{\prime}, \gamma} f\right)(x) \\
=\frac{x^{-\delta}}{\Gamma(\gamma)} \int_{x}^{\infty}(t-x)^{\gamma-1} t^{-\delta^{\prime}} F_{3}\left(\delta, \delta^{\prime}, \beta, \beta^{\prime} ; \gamma ; 1-\frac{x}{t}, 1-\frac{t}{x}\right) f(t) d t \\
\quad\left(\delta, \delta^{\prime}, \beta, \beta^{\prime}, \gamma \in \mathbf{C} \text { and } x>0 ; \operatorname{Re}(\gamma)>0\right) .
\end{gathered}
$$


and fractional derivative operators are defined by

$$
\left(D_{0+}^{\delta, \delta^{\prime}, \beta, \beta^{\prime}, \gamma} f\right)(x)=\left(I_{0+}^{-\delta,-\delta^{\prime},-\beta,-\beta^{\prime}, \gamma} f\right)(x)
$$

and

$$
\left(D_{0-}^{\delta, \delta^{\prime}, \beta, \beta^{\prime}, \gamma} f\right)(x)=\left(I_{0-}^{-\delta,-\delta^{\prime},-\beta,-\beta^{\prime}, \gamma} f\right)(x),
$$

where $F_{3}$ is the third Appell function (also known as the Horn function) $[14]$

$F_{3}\left(\delta, \delta^{\prime}, \beta, \beta^{\prime} ; \gamma ; x, y\right)=\sum_{m, n=0}^{\infty} \frac{(\delta)_{m}\left(\delta^{\prime}\right)_{n}(\beta)_{m}\left(\beta^{\prime}\right)_{n}}{(\gamma)_{m+n}} \frac{x^{m}}{m !} \frac{y^{n}}{n !}, \quad \max \{|x|,|y|\}<1$

The formula of generalized fractional integrals (3.1) and (3.2), and derivatives (3.3) and (3.4) of the power function are given below

$(3.6)\left(I_{0+}, z^{\prime}, 1\right)(x)$

$(3.6)=\frac{\Gamma(\rho) \Gamma\left(\rho+\gamma-\delta-\delta^{\prime}-\beta\right) \Gamma\left(\rho+\beta^{\prime}-\delta^{\prime}\right)}{\Gamma\left(\rho+\beta^{\prime}\right) \Gamma\left(\rho+\gamma-\delta-\delta^{\prime}\right) \Gamma\left(\rho+\gamma-\delta^{\prime}-\beta\right)} x^{\rho+\gamma-\delta-\delta^{\prime}-1}$

$\left(\operatorname{Re}(\gamma)>0, \operatorname{Re}(\rho)>\max \left\{0, \operatorname{Re}\left(\delta+\delta^{\prime}+\beta-\gamma\right),\left(\delta^{\prime}-\beta^{\prime}\right)\right\}, x>0\right)$

$\left(I_{0-}^{\delta, \delta^{\prime}, \beta, \beta^{\prime}, \gamma} z^{\rho-1}\right)(x)$

$=\frac{\Gamma\left(1-\rho-\gamma+\delta+\delta^{\prime}\right) \Gamma\left(1-\rho+\delta+\beta^{\prime}-\gamma\right) \Gamma(1-\rho+\beta)}{\Gamma(1-\rho) \Gamma\left(1-\rho+\delta+\delta^{\prime}+\beta+\beta^{\prime}-\gamma\right) \Gamma(1-\rho+\delta-\beta)} x^{\rho+\gamma-\delta-\delta^{\prime}-1}$

$\left(\operatorname{Re}(\gamma)>0, \operatorname{Re}(\rho)<1+\max \left\{0, \operatorname{Re}(-\beta), \operatorname{Re}\left(\delta+\delta^{\prime}-\gamma\right), \operatorname{Re}\left(\delta+\beta^{\prime}-\gamma\right)\right\}, x>0\right)$

$$
\left(D_{0+}^{\delta, \delta^{\prime}, \beta, \beta^{\prime}, \gamma} z^{\rho-1}\right)(x)
$$

$=\frac{\Gamma(\rho) \Gamma(\rho+\delta-\beta) \Gamma\left(\rho+\delta+\delta^{\prime}+\beta^{\prime}-\gamma\right)}{\Gamma(\rho-\beta) \Gamma\left(\rho+\delta-\delta^{\prime}-\gamma\right) \Gamma\left(\rho+\delta+\beta^{\prime}-\gamma\right)} x^{\rho+\delta+\delta^{\prime}-\gamma-1}$

$\left(\operatorname{Re}(\gamma)>0, \operatorname{Re}(\rho)>\max \left\{0, \operatorname{Re}(-\delta+\beta), \operatorname{Re}\left(-\delta-\delta^{\prime}-\beta^{\prime}+\gamma\right)\right\}, x>0\right)$

$$
\begin{aligned}
& \left(D_{0-}^{\delta, \delta^{\prime}, \beta, \beta^{\prime}, \gamma} z^{-\rho}\right)(x) \\
& =\frac{\Gamma\left(-\delta-\delta^{\prime}+\gamma+\rho\right) \Gamma\left(-\delta^{\prime}-\beta+\gamma+\rho\right) \Gamma\left(\beta^{\prime}+\rho\right)}{\Gamma(\rho) \Gamma\left(-\delta-\delta^{\prime}-\beta+\gamma+\rho\right) \Gamma\left(-\delta^{\prime}+\beta^{\prime}+\rho\right)} x^{\delta+\delta^{\prime}-\gamma-\rho}
\end{aligned}
$$

$\left(\operatorname{Re}(\gamma)>0, \operatorname{Re}(\rho)>\max \left\{\operatorname{Re}\left(-\beta^{\prime}\right), \operatorname{Re}\left(\delta^{\prime}+\beta-\gamma\right)+[\operatorname{Re}(\gamma)]+1\right\}, x>0\right)$ 
Theorem 3. If $x>0,0<\alpha \leq 1,\left\{\delta, \delta^{\prime}, \beta, \beta^{\prime}, \gamma, a_{i}, b_{j}\right\} \subset C, i=1, \ldots, p, j=$ $1, \ldots, q, \operatorname{Re}(\gamma)>0,1+\min \left\{\operatorname{Re}\left(\gamma-\delta-\delta^{\prime}-\beta\right), \operatorname{Re}\left(\gamma-\delta-\delta^{\prime}\right), \operatorname{Re}\left(\beta^{\prime}\right)\right\}>0$, then

$$
\begin{aligned}
& \left(I_{0+}^{\delta, \delta^{\prime}, \beta, \beta^{\prime}, \gamma}\left[{ }_{p}^{\alpha} F_{q}(z)\right]\right)(x)=x^{\gamma-\delta-\delta^{\prime}} \frac{\Gamma\left(\gamma-\delta^{\prime}-\beta+1\right) \Gamma\left(\gamma-\delta-\delta^{\prime}+1\right) \Gamma\left(\beta^{\prime}+1\right)}{\Gamma\left(\gamma-\delta-\delta^{\prime}-\beta+1\right) \Gamma\left(\beta^{\prime}-\delta^{\prime}+1\right)} \\
& \underset{p+3}{\alpha} F_{q+3}\left[\begin{array}{c}
1, \gamma-\delta-\delta^{\prime}-\beta+1, \beta^{\prime}-\delta^{\prime}+1, a_{1}, \ldots, a_{p} ; \\
\beta^{\prime}+1, \gamma-\delta^{\prime}-\beta+1, \gamma-\delta-\delta^{\prime}+1, b_{1}, \ldots, b_{q} ;
\end{array}\right],
\end{aligned}
$$

and if $x>0,0<\alpha \leq 1,\left\{\delta, \delta^{\prime}, \beta, \beta^{\prime}, \gamma, a_{i}, b_{j}\right\} \subset C, i=1, \ldots, p, j=$ $1, \ldots, q, \operatorname{Re}(\gamma)>0,1+\min \left\{\operatorname{Re}\left(\delta-\delta^{\prime}-\gamma\right), \operatorname{Re}\left(\delta+\beta^{\prime}-\gamma\right), \operatorname{Re}(-\beta)\right\}>0$, then

$$
\begin{aligned}
& \left(I_{0-}^{\delta, \delta^{\prime}, \beta, \beta^{\prime}, \gamma}\left[\frac{1}{z} F_{q} F_{q}\left(\frac{1}{z}\right)\right]\right)(x)=\frac{\Gamma\left(1-\gamma+\delta+\delta^{\prime}\right) \Gamma\left(1+\delta+\beta^{\prime}-\gamma\right) \Gamma(1-\beta)}{\Gamma\left(1+\delta+\delta^{\prime}+\beta+\beta^{\prime}-\gamma\right) \Gamma(1+\delta-\beta)} \\
& x_{p+\delta}^{\gamma-\delta-\delta^{\prime}-1{ }_{p+3}^{\alpha} F_{q+3}}\left[\begin{array}{l}
1-\gamma+\delta+\delta^{\prime}, 1+\delta+\beta^{\prime}-\gamma, 1-\beta, a_{1}, \ldots, a_{p} ; \\
1+\delta+\delta^{\prime}+\beta+\beta^{\prime}-\gamma, 1+\delta-\beta, 1, b_{1}, \ldots, b_{q} ;
\end{array}\right] .
\end{aligned}
$$

Proof. The hypergeometric function with $p$ numerator and $q$ denominator parameters is defined by [1]

$$
{ }_{p} F_{q}\left[\begin{array}{c}
a_{1}, \ldots a_{p} ; \\
b_{1}, \ldots b_{q} ;
\end{array}\right]=\sum_{n=0}^{\infty} \frac{\left(a_{1}\right)_{n} \ldots\left(a_{p}\right)_{n}}{\left(b_{1}\right)_{n} \ldots\left(b_{q}\right)_{n}} \frac{z^{n}}{n !} .
$$

From (3.13) and (3.6), we can write

$$
\begin{aligned}
& \left(I_{0-}^{\delta, \delta^{\prime}, \beta, \beta^{\prime}, \gamma}\left[{ }_{p}^{\alpha} F_{q}(z)\right]\right)(x)=\sum_{n=0}^{\infty} \frac{\left(a_{1}\right)_{n, \alpha}\left(a_{2}\right)_{n} \ldots\left(a_{p}\right)_{n}}{\left(b_{1}\right)_{n} \ldots\left(b_{q}\right)_{n} n !} \\
& \cdot\left(\frac{\Gamma\left(\gamma-\delta-\delta^{\prime}-\beta+n+1\right) \Gamma\left(\beta^{\prime}-\delta^{\prime}+n+1\right) \Gamma(n+1)}{\Gamma\left(\gamma-\delta-\delta^{\prime}+n+1\right) \Gamma\left(\gamma-\delta^{\prime}-\beta+n+1\right) \Gamma\left(\beta^{\prime}+n+1\right)} x^{\gamma-\delta-\delta^{\prime}+n}\right) \\
& =x^{\gamma-\delta-\delta^{\prime}} \frac{\Gamma\left(\gamma-\delta^{\prime}-\beta+1\right) \Gamma\left(\gamma-\delta-\delta^{\prime}+1\right) \Gamma\left(\beta^{\prime}+1\right)}{\Gamma\left(\gamma-\delta-\delta^{\prime}-\beta+1\right) \Gamma\left(\beta^{\prime}-\delta^{\prime}+1\right)} \\
& \sum_{n=0}^{\infty} \frac{(1)_{n}\left(\gamma-\delta-\delta^{\prime}-\beta+1\right)_{n}\left(\beta^{\prime}-\delta^{\prime}+1\right)_{n}}{\left(\beta^{\prime}+1\right)_{n}\left(\gamma-\delta-\delta^{\prime}+1\right)_{n}\left(\gamma-\delta^{\prime}-\beta+1\right)_{n}} \frac{\left(a_{1}\right)_{n, \alpha}\left(a_{2}\right)_{n} \ldots\left(a_{p}\right)_{n}}{\left(b_{1}\right)_{n} \cdots\left(b_{q}\right)_{n}} \frac{x^{n}}{n !},
\end{aligned}
$$

which in view of definition (3.13), is the right hand side of assertion (3.12).

In the same way, using (3.13) and applying the formula (3.7), we can derive the assertion (3.12). 
Theorem 4. If $x>0,0<\alpha \leq 1,\left\{\delta, \delta^{\prime}, \beta, \beta^{\prime}, \gamma, a_{i}, b_{j}\right\} \subset C, i=1, \ldots, p, j=$ $1, \ldots, q, \operatorname{Re}(\gamma)>0,1+\min \left\{\operatorname{Re}(\delta-\beta), \operatorname{Re}\left(\delta+\delta^{\prime}+\beta^{\prime}-\gamma\right)\right\}>0$, then $\left(D_{0+}^{\delta, \delta^{\prime}, \beta, \beta^{\prime}, \gamma}\left[{ }_{p}^{\alpha} F_{q}(z)\right]\right)(x)=x^{\delta+\delta^{\prime}-\gamma} \frac{\Gamma(1+\delta-\beta) \Gamma\left(1+\delta+\delta^{\prime}+\beta^{\prime}-\gamma\right)}{\Gamma(1-\beta) \Gamma\left(1+\delta-\delta^{\prime}-\gamma\right) \Gamma\left(1+\delta+\beta^{\prime}-\gamma\right)}$

$$
{ }_{p+3}^{\alpha} F_{q+3}\left[\begin{array}{c}
1,1+\delta-\beta, 1+\delta+\delta^{\prime}+\beta^{\prime}-\gamma, a_{1}, \ldots, a_{p} ; \\
1-\beta, 1+\delta-\delta^{\prime}-\gamma, 1+\delta+\beta^{\prime}-\gamma, b_{1}, \ldots, b_{q} ;
\end{array}\right],
$$

and if $x>0,0<\alpha \leq 1,\left\{\delta, \delta^{\prime}, \beta, \beta^{\prime}, \gamma, a_{i}, b_{j}\right\} \subset C, i=1, \ldots, p, j=$ $1, \ldots, q, \operatorname{Re}(\gamma)>0,1+\min \left\{\operatorname{Re}\left(-\delta-\delta^{\prime}+\gamma\right), \operatorname{Re}\left(-\delta^{\prime}-\beta+\gamma\right), \operatorname{Re}\left(\beta^{\prime}\right)\right\}>0$, then

$$
\begin{aligned}
& \left(D_{0-}^{\delta, \delta^{\prime}, \beta, \beta^{\prime}, \gamma}\left[\frac{1}{z} F_{q} F_{q}\left(\frac{1}{z}\right)\right]\right)(x)=\frac{\Gamma\left(-\delta-\delta^{\prime}+\gamma+1\right) \Gamma\left(-\delta^{\prime}-\beta+\gamma+1\right) \Gamma\left(\beta^{\prime}+1\right)}{\Gamma\left(-\delta-\delta^{\prime}-\beta+\gamma+1\right) \Gamma\left(-\delta^{\prime}+\beta^{\prime}+1\right)} \\
& x^{\delta+\delta^{\prime}-\gamma-1} \underset{p+3}{\alpha} F_{q+3}\left[\begin{array}{c}
-\delta-\delta^{\prime}+\gamma+1,-\delta^{\prime}-\beta+\gamma+1, \beta^{\prime}+1, a_{1}, \ldots, a_{p} ; \\
-\delta-\delta^{\prime}-\beta+\gamma+1,-\delta^{\prime}+\beta^{\prime}+1,1, b_{1}, \ldots, b_{q} ;
\end{array}\right] .
\end{aligned}
$$

Proof. From (3.13) and (3.8), we get

$$
\begin{aligned}
& \left(D_{0+}^{\delta, \delta^{\prime}, \beta, \beta^{\prime}, \gamma}\left[{ }_{p}^{\alpha} F_{q}(z)\right]\right)(x)=\sum_{n=0}^{\infty} \frac{\left(a_{1}\right)_{n, \alpha}\left(a_{2}\right)_{n} \ldots\left(a_{p}\right)_{n}}{\left(b_{1}\right)_{n} \ldots\left(b_{q}\right)_{n} n !} \\
& \cdot\left(\frac{\Gamma(n+1) \Gamma(\delta-\beta+n+1) \Gamma\left(\delta+\delta^{\prime}+\beta^{\prime}-\gamma+n+1\right)}{\Gamma(-\beta+n+1) \Gamma\left(\delta-\delta^{\prime}-\gamma+n+1\right) \Gamma\left(\delta+\beta^{\prime}-\gamma+n+1\right)} x^{\delta+\delta^{\prime}-\gamma+n}\right) \\
& \quad=x^{\delta+\delta^{\prime}-\gamma} \frac{\Gamma(1+\delta-\beta) \Gamma\left(1+\delta+\delta^{\prime}+\beta^{\prime}-\gamma\right)}{\Gamma(1-\beta) \Gamma\left(1+\delta-\delta^{\prime}-\gamma\right) \Gamma\left(1+\delta+\beta^{\prime}-\gamma\right)} \\
& \sum_{n=0}^{\infty} \frac{(1)_{n}(1+\delta-\beta)_{n}\left(1+\delta+\delta^{\prime}+\beta^{\prime}-\gamma\right)_{n}}{(1-\beta)_{n}\left(1+\delta-\delta^{\prime}-\gamma\right)_{n}\left(1+\delta+\beta^{\prime}-\gamma\right)_{n}} \frac{\left(a_{1}\right)_{n, \alpha}\left(a_{2}\right)_{n} \ldots\left(a_{p}\right)_{n}}{\left(b_{1}\right)_{n} \ldots\left(b_{q}\right)_{n}} \frac{x^{n}}{n !},
\end{aligned}
$$

By using (3.13) again, we can reach to (3.14).

Similarly, using formula (3.9), assertion (3.15) can be derived.

Remark 1. If we take $\alpha=1$ in the above mentioned theorems, we get the corresponding results for the classical hypergeometric functions ${ }_{2} F_{1}$ and ${ }_{p} F_{q}$.

\section{Acknowledgement}

This work was partially supported by SERB, Govt. of India, Research Project No. EMR/2016/000351, sanctioned to the author RKJ. The authors are grateful to the anonymous referee(s) for their valuable suggestions, which resulted in the improvement of the paper. 


\section{References}

[1] E. Arthur, W. Magnus, F. Oberhettinger, F. Tricomi, and H. Bateman, Higher transcendental functions, vol. 1. New York, NY: McGraw-Hill, 1953.

[2] R. Gorenflo, A. Kilbas, F. Mainardi, and S. Rogosin, Mittag-Leffler Functions, Related Topics and Applications. Berlin, Heidelberg: Springer, 2014, doi: 10.1007/978-3-662-43930-2.

[3] G. Jumarie, "On the representation of fractional Brownian motion as an integral with respect to (dt)a", Applied Mathematics Letters, vol. 18, no. 7, pp. 739-748, Jul. 2005, doi: 10.1016/j.aml.2004.05.014.

[4] G. Jumarie, "Modified Riemann-Liouville derivative and fractional Taylor series of nondifferentiable functions further results", Computers \& Mathematics with Applications, vol. 51, no. 9-10, pp. 1367-1376, May 2006, doi: 10.1016/j.camwa.2006.02.001.

[5] G. Jumarie, "Table of some basic fractional calculus formulae derived from a modified Riemann-Liouville derivative for non-differentiable functions", Applied Mathematics Letters, vol. 22, no. 3, pp. 378-385, Mar. 2009, doi: 10.1016/j.aml.2008.06.003.

[6] G. Jumarie, "Laplace's transform of fractional order via the MittagLeffler function and modified Riemann-Liouville derivative", Applied Mathematics Letters, vol. 22, no. 11, pp. 1659-1664, Nov. 2009, doi: 10.1016/j.aml.2009.05.011.

[7] G. Jumarie, "Cauchy's integral formula via the modified Riemann-Liouville derivative for analytic functions of fractional order", Applied Mathematics Letters, vol. 23, no. 12, pp. 1444-1450, Dec. 2010, doi: 10.1016/j.aml.2010.08.001.

[8] G. Jumarie, "Fractional Euler's integral of first and second kinds. Application to fractional Hermite's polynomials and to probability density of fractional order", Journal of Applied Mathematics and Informatics, vol. 28, no. 1-2, pp. 257-273, 2010. [On line]. Available: http://bit.ly/2ZHWi1H

[9] G. Jumarie, "The Leibniz rule for fractional derivatives holds with nondifferentiable functions", Mathematics and Statistics, vol. 1, no. 2, pp. 50-52, 2013. [On line]. Available: http://bit.ly/20PTb71

[10] G. Jumarie, Fractional differential calculus for non-differentiable functions: mechanics, geometry, stochastics, information theory. Saarbrucken: LAP LAMBERT Academic Publishing, 2013. 
[11] G. Jumarie, "On the derivative chain-rules in fractional calculus via fractional difference and their application to systems modelling", Central European Journal of Physics, vol. 11, no. 6, pp. 617-633, Oct. 2014, doi: 10.2478/s11534-013-0256-7.

[12] E. Rainville, Special Functions, New York, NY :The Macmillan Company, 1960.

[13] M. Saigo and N. Maeda, "More generalization of fractional calculus", in Transform methods \& special functions, Varna '96: second international workshop : proceedings, 1998, pp. 386-400.

[14] H. Srivastava and P. Karlsson, Multiple gaussian hypergeometric series. Chichester: Horwood, 1985.

[15] N. Virchenko, "On the generalized confluent hypergeometric function and its applications", Fractional Calculus and Applied Analysis, vol. 9, no. 2, pp. 101-108, 2006. [On line]. Available: http://bit.ly/20KSD1V

[16] N. Virchenko, "On some generalizations of classical integral transforms". Mathematica. Balkanica, vol. 26, no. 1-2, 2012. [On line]. Available: http://bit.ly/2MUiLVJ 